\title{
Quantum Hall effect in single wide quantum wells
}

\author{
M. Abolfath ${ }^{1,3}$ * L. Belkhir ${ }^{1,2}$, and N. Nafari ${ }^{3}$ \\ ${ }^{1}$ Department of Physics, Indiana University, Bloomington, Indiana 47405 \\ ${ }^{2}$ Xerox Corporation, 800 Phillips Road, 147-59B, Webster, NY 14580 \\ ${ }^{3}$ Center for theoretical Physics and Mathematics, Atomic Energy Organization of Iran, P.O.Box \\ 11365-8486, Tehran, Iran
}

(May 26, 2022)

\begin{abstract}
We study the quantum Hall states in the lowest Landau level for a single wide quantum well. Due to a separation of charges to opposite sides of the well, a single wide well can be modelled as an effective two level system. We provide numerical evidence of the existence of a phase transition from an incompressible to a compressible state as the electron density is increased for specific well width. Our numerical results show a critical electron density which depends on well width, beyond which a transition incompressible double layer quantum Hall state to a mono-layer compressible state occurs. We also calculate the related phase boundary corresponding to destruction of the collective mode energy gap. We show that the effective tunneling term and the interlayer separation are both renormalised by the strong magnetic field. We also exploite the local density functional techniques in the presence of strong magnetic field at $\nu=1$ to calculate renormalized $\Delta_{S A S}$. The numerical results shows good
\end{abstract}

*email: abolfath@gibbs.physics.indiana.edu 
agreement between many-body calculations and local density functional techniques in the presence of a strong magnetic field at $\nu=1$. we also discuss implications of this work on the $\nu=1 / 2$ incompressible state observed in SWQW.

Typeset using REVTEX 


\section{INTRODUCTION}

Recent interest in the properties of high-mobility quasi three-dimensional electron systems [1,2] and double quantum well structures (DQWs) in strong magnetic fields [3, [4] which are quite different in their structural details, has led to discovery of a novel forms of the fractional quantum Hall effect. In the study by Eisenstein et al. [4, the sample is a traditional symmetric double quantum well (DQWs) structure. The envelope wave function in the middle of the sample is small because there is a large barrier between the two wells, whereas in the work of Suen et al. [1,2] the samples are actually a single wide quantum wells (SWQWs). The self-consistent electric field arising from the presence of the electrons themselves, splits the well into two spatially separated electron layers in the z-direction, effectively creating a double layer structure, having a small self-consistent barrier with a finite envelope function in the middle.

Based on the fact that these samples are single wide quantum wells, it has been argued by some authors that the quantum Hall mechanism of these systems is different from DQWs. However, it has been claimed by Song He et al. [5] that the result of finite size exact diagonalization calculation disagrees with this viewpoint and the bare $\Delta_{S A S}$, the difference between the second and first subband energy in zero magnetic field, which is reported by Ref. [2] is not the relevant parameter determining the nature of the ground state in a strongly correlated system. One may expect that the electronic structure of a SWQW is affected by a strong magnetic field and therefore it changes the $\Delta_{S A S}$. In other words $\Delta_{S A S}$ is renormalized by a strong magnetic field. It has been claimed that the renormalized $\Delta_{S A S}^{B}$ is small enough for the Halperin state [6], to be the ground state [5]. In this article we report the

numerical calculation of the renormalized $\Delta_{S A S}^{B}$ for different realistic models of the samples in Ref. [2]. Our analytical expression for renormilized tunneling at $\nu=1$ is equivalent with the results of Hartree-Fock approximation (HFA). We also implicate the renormilized tunneling's difinition for fractional filling factors e.g. $\nu=1 / 2$. Our first model is a many body Hamiltonian calculation with electron-electron, isospin-potential interactions, where 
one may think of the layer index as an isospin index and the second is based on the local density functional approximation (LDFA) in the presence of a strong magnetic field. We also use LDFA to evaluate the bare and renormalized tunneling at filling factor $\nu=1$. There are some special cases in which SWQWs may effectively described by DQWs, i.e. high areal electron density and large well width. In this article DQW is the jargon for SWQW at high density or large width where the overlap of wave functions is negligible in the middle of the well. In these limits the renormalized $\Delta_{S A S}^{B}$, which is the difference energy between the first and second subbands, in the presence of a strong perpendicular magnetic field, is small enough and the quantum Hall ground states may be described by Halperin's wave functions. In the past few years SWQW's have been approximated by DQW, but there are significant correction to the DQW Hamiltonian due to overlap of electrons wavefunction which are localized in the SWQWs edges. In this article, we show that these corrections become more important either by decreasing the electron density or decreasing the well width. We also show that the two-component quantum Hall ground state at $\nu=1$ which is stabilized by a small $\Delta_{S A S}^{B}$ at high $N_{s}$ may evolve continuously to a one-component normal state at large $\Delta_{S A S}^{B}$ and low $N_{s}$ where we call a state of the 2DES with no quantum Hall effects by normal state.

Throughout this paper we neglect any explicit consideration of electron spin, assuming that the magnetic field $B$ is always high enough to totally spin polarize the system due to a large Zeeman splitting. We also ignore any effect of Landau level mixing and higher subbands, working exclusively in the lowest spin-split Landau level of the lowest subband. These assumptions are consistent with all the specific experiments to be disscused in this paper. It is obvious that the DQW and SWQW have some common properties which bring them into the same class. For the sake of simplicity, we use the term of bilayer electron systems (BLES) to unified DQW and SWQW as long as we concern about their common properties. There are two energy scales associated with a BLES in the presence of strong magnetic field, $\Delta_{S A S}^{B}$ and the many-body Coulomb interaction between electrons $e^{2} / \epsilon_{0} \ell_{0}^{2}$ where $\epsilon_{0}$ is 
dielectric constant and $\ell_{0}=\sqrt{\hbar c / e B}$ is the magnetic length corresponding to cyclotron orbit radius with electron cyclotron energy $\hbar \omega_{c}$, where $\omega_{c}=e B / m^{*} c$ is the cyclotron frequency. Competition between these two energies leads to a rich phase diagram. In a single layer twodimensional electron system (2DES), the integer quantum Hall effect (IQHE) is related to gaps in the single particle density of states produced by the electron cyclotron energy $\left(\hbar \omega_{c}\right)$, while in the fractional quantum Hall effect (FQHE), the gap in the excitation spectrum is the result of the electrons' Coulomb interaction. The quantum Hall plateau for BLES is controlled by the total filling factor $\left(\nu_{t o t}\right)$ which is the sum of the filling factors in each layer. Therefore the odd-IQHE of BLES corresponds to even denominator filling factor for each layer, for example $\nu_{t o t}=1$ is produced by $\nu=1 / 2$ in each layer, even though $\nu=1 / 2$ in a single layer does not yield a plateau. Observation of odd-IQHE is due to correlation between electrons in different layers which is destroyed if they are uncorrelated by increasing the layer distance or decreasing $\Delta_{S A S}^{B}$, although for DQW with small enough separations the IQHE survives even in the limit $\Delta_{S A S}^{B} \rightarrow 0$, due to a spontanous broken symmetry. This phase transtion has been observed for DQW by Boebinger et al. [3] and explained theoretically by MacDonald et al. [7]. This effect has also been observed for SWQW by Suen et al. [1]. In this article we present a theoretical study of SWQW incompressible to compressible phase transitions at filling factor $\nu=1$.

In Section [1], we obtain the many body Hamiltonian of SWQWs in the absence of magnetic field. We study the effect of strong magnetic field in Section [II] by projection of Hamiltonian onto the lowest Landaue level's Hilbert space and comparing with the HartreeFock approximation at $\nu=1$. In Section $\mathbb{I V}$, we use local density functional approximation in the presence of perpendicular strong magnetic field to find SWQWs band structure to evaluate the renormalized $\Delta_{S A S}$ and comparing with other results. In Section $\nabla$, the collective modes in quantum Hall state is studied. We present a phase diagram for quantum Hall compressible-incompressible phase transition and comparing with experimental results. 


\section{MANY BODY HAMILTONIAN OF SINGLE WIDE QUANTUM WELL IN ZERO MAGNETIC FIELD}

In a SWQW the electrons are confined in the $x-y$ plane by an external potential barrier and therfore the energy spectrum becomes quantized into electric subbands. The number of filled subbands is a function of the areal density, $N_{s}$. In experimental work at zero magnetic field, the first two subbands are typically filled [1,2]. Neglecting high subbands, the subband degree of freedom SWQWs effectively reduces to a two level system. This system may mapped to a 2D electron system with an isospin degree of freedom. In the isospin language the isospin states, up $(\uparrow)$ and down $(\downarrow)$ refer to first and second subbands respectively. In the absence of perpindicular electric field bias, the system is balanced and the up (down) states are reflection symmetric (antisymmetric) states respectively, with bare eigenenergy difference $\Delta_{S A S}^{0}$. For the sake of simplicity we define $\Delta_{S A S}$ as the eigenenergy differece between the two subbands in both balanced and imbalanced cases. The general second quantized many body hamiltonian in this picture is

$$
\hat{H}=\hat{T}+\hat{V}
$$

where $\hat{T}$, in analogy to the double well case is refered to as the tunneling energy

$$
\hat{T}=-\frac{\Delta_{S A S}^{0}}{2} \sum_{\mathbf{p}}\left(\hat{C}_{\mathbf{p}}^{\dagger \uparrow} \hat{C}_{\mathbf{p}}^{\uparrow}-\hat{C}_{\mathbf{p}}^{\dagger \downarrow} \hat{C}_{\mathbf{p}}^{\downarrow}\right)
$$

Here $\Delta_{S A S}^{0}$ is the subband energy difference of noninteracting electron system in the SWQW. The interaction part of the many-body Hamiltonian employed in our studies can be written in zero magnetic field as

$$
\hat{V}=\frac{1}{2} \sum_{\mathbf{p}, \mathbf{p}^{\prime}, \mathbf{q}} \sum_{\{\sigma\}} V_{\mathbf{q}}^{\sigma_{1}^{\prime} \sigma_{2}^{\prime} \sigma_{1} \sigma_{2}} \hat{C}_{\mathbf{p}+\mathbf{q}}^{\dagger \sigma_{1}^{\prime}} \hat{C}_{\mathbf{p}^{\prime}-\mathbf{q}}^{\dagger \sigma_{2}^{\prime}} \hat{C}_{\mathbf{p}^{\prime}}^{\sigma_{2}} \hat{C}_{\mathbf{p}}^{\sigma_{1}}
$$

In the above equation $V_{\mathbf{q}}^{\sigma_{1}^{\prime} \sigma_{2}^{\prime} \sigma_{1} \sigma_{2}}$ is the Fourier transform of the electron-electron Coulomb interaction

$$
V_{\mathbf{q}}^{\sigma_{1}^{\prime} \sigma_{2}^{\prime} \sigma_{1} \sigma_{2}}=\frac{2 \pi e^{2}}{\epsilon_{0} q} \int d z_{1} \int d z_{2} \psi^{* \sigma_{1}^{\prime}}\left(z_{1}\right) \psi^{* \sigma_{2}^{\prime}}\left(z_{2}\right) \psi^{\sigma_{1}}\left(z_{1}\right) \psi^{\sigma_{2}}\left(z_{2}\right) e^{-q\left|z_{1}-z_{2}\right|}
$$


where $\mathbf{q}$ is the in plane wavevector. $\hat{C}_{\mathbf{p}}^{\dagger \sigma}\left(\hat{C}_{\mathbf{p}}^{\sigma}\right)$ is the creation (anihilation) operator for electrons with 2D momentum $\mathbf{p}$ and isospin state $\sigma$ and obeys,

$$
\left\{\hat{C}_{\mathbf{p}}^{\sigma}, \hat{C}_{\mathbf{p}^{\prime}}^{\dagger \sigma^{\prime}}\right\}=\delta_{\mathbf{p}, \mathbf{p}^{\prime}} \delta^{\sigma, \sigma^{\prime}}
$$

It is convenient to define the 4 -vector isospin density operator

$$
\begin{gathered}
\sigma_{\mathbf{k}}^{\mu}=\left(\sigma_{\mathbf{k}}^{0}, \sigma_{\mathbf{k}}^{a}\right), \\
\sigma_{\mathbf{k}}^{a}=\left(\sigma_{\mathbf{k}}^{x}, \sigma_{\mathbf{k}}^{y}, \sigma_{\mathbf{k}}^{z}\right),
\end{gathered}
$$

where $\sigma^{0}$ is unit matrix and $\sigma^{a}$ is the ath component of Pauli matrices

$$
\sigma^{x}=\left(\begin{array}{cc}
0 & 1 \\
1 & 0
\end{array}\right), \quad \sigma^{y}=\left(\begin{array}{cc}
0 & -i \\
i & 0
\end{array}\right), \quad \sigma^{z}=\left(\begin{array}{cc}
1 & 0 \\
0 & -1
\end{array}\right) .
$$

Here $\mu \in\{0,1,2,3\}$. The zeroth component of $\sigma_{\mathbf{k}}^{\mu}$ is the scalar density operator and the rest is the vectorial isospin density. One may write the 4-vector isospin density operator in terms of creation-annihilation operators

$$
\hat{\sigma}_{\mathbf{q}}^{\mu}=\sum_{\mathbf{p}}\left(\begin{array}{ll}
\hat{C}_{\mathbf{p}-\mathbf{q}}^{\dagger \uparrow} & \hat{C}_{\mathbf{p}-\mathbf{q}}^{\dagger \downarrow}
\end{array}\right) \sigma^{\mu}\left(\begin{array}{c}
\hat{C}_{\mathbf{p}}^{\uparrow} \\
\hat{C}_{\mathbf{p}}^{\downarrow}
\end{array}\right)
$$

The transformed hamiltonian Eq.(1) with continous wave vector $\mathbf{q}$ simplifies to

$$
\hat{H}=-\frac{\Delta_{S A S}}{2} \sigma^{z}(\mathbf{q}=0)+\frac{1}{2} \int \frac{d^{2} \mathbf{q}}{(2 \pi)^{2}} \sigma_{\mu}(-\mathbf{q}) V^{\mu \nu}(\mathbf{q}) \sigma_{\nu}(\mathbf{q}) \text {. }
$$

The isospin potential $V^{\mu \nu}(\mathbf{q})$ in Eq.(9) describes the electron-electron isospin interaction, for the sake of simplicity we call the $V^{\mu \nu}(\mathbf{q})$, the isopotential matrix elements. They are linear combinations of the coulomb potential form factors in Eq.(4) and are defined in Eq.(11).

One finds after a little algebraic calculation, $\Delta_{S A S}^{0}$ is renormalized by the exchange electron-electron interaction

$$
\Delta_{S A S}=\Delta_{S A S}^{0}+2 \int \frac{d^{2} \mathbf{q}}{(2 \pi)^{2}} V_{0 z}(\mathbf{q})
$$

The enhancent may be positive or negative, depending on the sign of integral upon $V_{0 z}(\mathbf{q})$. For example it is positive if the density is low and negative at high density for a given well 
width. Therefore for large (small) bare $\Delta_{S A S}^{0}$ the renormalized $\Delta_{S A S}$ is greater (lesser) than $\Delta_{S A S}^{0}$ respectively. In the section $\mathbb{\mathbb { V }}$, we will calculate $\Delta_{S A S}$ by local density functional approximation (LDFA) where the effect of local exchange-correlation interaction is included in $\Delta_{S A S}$. One may show that the results of LDFA and HFA are close, hence the left hand side of Eq.(10) can be evaluated by LDFA.

The non-zero isopotentials elements in Eq.(9) may be written in terms of the electronelectron interaction of matrix elements defined in Eq.(任).

$$
\begin{gathered}
V_{00}(\mathbf{q})=\frac{1}{4}\left(V_{\mathbf{q}}^{\uparrow \uparrow}+2 V_{\mathbf{q}}^{\uparrow \downarrow}+V_{\mathbf{q}}^{\downarrow \downarrow}\right), \\
V_{z z}(\mathbf{q})=\frac{1}{4}\left(V_{\mathbf{q}}^{\uparrow \uparrow}-2 V_{\mathbf{q}}^{\uparrow \downarrow}+V_{\mathbf{q}}^{\downarrow \downarrow}\right), \\
V_{0 x}(\mathbf{q})=V_{x 0}(\mathbf{q})=\frac{1}{2}\left(C_{\mathbf{q}}^{\uparrow \downarrow}+D_{\mathbf{q}}^{\uparrow \downarrow}\right), \\
V_{0 z}(\mathbf{q})=V_{z 0}(\mathbf{q})=\frac{1}{4}\left(V_{\mathbf{q}}^{\uparrow \uparrow}-V_{\mathbf{q}}^{\downarrow \downarrow}\right), \\
V_{x z}(\mathbf{q})=V_{z x}(\mathbf{q})=\frac{1}{2}\left(C_{\mathbf{q}}^{\uparrow \downarrow}-D_{\mathbf{q}}^{\uparrow \downarrow}\right), \\
V_{x x}(\mathbf{q})=B_{\mathbf{q}}^{\uparrow \downarrow},
\end{gathered}
$$

Taking the advantage of symmetry properties of Eq.(19), and the fact that the subband eigenfunctions are real, we define

$$
\begin{gathered}
V_{\mathbf{q}}^{\uparrow \uparrow} \equiv V_{\mathbf{q}}^{\uparrow \uparrow \uparrow}, \quad V_{\mathbf{q}}^{\downarrow \downarrow} \equiv V_{\mathbf{q}}^{\downarrow \downarrow \downarrow \downarrow}, \\
V_{\mathbf{q}}^{\uparrow \downarrow} \equiv V_{\mathbf{q}}^{\uparrow \downarrow \uparrow \downarrow}=V_{\mathbf{q}}^{\downarrow \uparrow \downarrow \uparrow}, \\
B_{\mathbf{q}}^{\uparrow \downarrow} \equiv V_{\mathbf{q}}^{\uparrow \uparrow \downarrow}=V_{\mathbf{q}}^{\downarrow \uparrow \uparrow \downarrow}=V_{\mathbf{q}}^{\uparrow \downarrow \downarrow \uparrow}=V_{\mathbf{q}}^{\downarrow \downarrow \uparrow \uparrow}, \\
C_{\mathbf{q}}^{\uparrow \downarrow} \equiv V_{\mathbf{q}}^{\uparrow \uparrow \uparrow \downarrow}=V_{\mathbf{q}}^{\uparrow \downarrow \uparrow}=V_{\mathbf{q}}^{\uparrow \downarrow \uparrow \uparrow}=V_{\mathbf{q}}^{\downarrow \uparrow \uparrow \uparrow}, \\
D_{\mathbf{q}}^{\uparrow \downarrow} \equiv V_{\mathbf{q}}^{\downarrow \downarrow \downarrow \uparrow}=V_{\mathbf{q}}^{\downarrow \downarrow \uparrow \downarrow}=V_{\mathbf{q}}^{\downarrow \uparrow \downarrow \downarrow}=V_{\mathbf{q}}^{\uparrow \downarrow \downarrow \downarrow} .
\end{gathered}
$$

Note that the $y$ component of isopotential are zero. In the next section we will see that these elements are still zero in the presence of the prependicular magnetic field. However, 
it has been found that [16] the $y$ component are not zero due to tilted magnetic field effect and therefore all of isopotential elements in Hamiltonian are nonzero. One may find by inversion symmetry of the Hamiltonian that both $C_{\mathbf{q}}^{\uparrow \downarrow}$ and $D_{\mathbf{q}}^{\uparrow \downarrow}$ are zero unless the system is imbalanced by applying a perpendicular external electric field.

Another extreme case may happen in the high density balanced regime, shown in Fig.(何). The overlap integral between the wavefunctions in the left and right is negligible and therefore we may show that, $\left|\psi^{\uparrow}(z)\right|=\left|\psi^{\downarrow}(z)\right|$. In this regime one finds the only nonzero matrix elements are $B_{\mathbf{q}}^{\uparrow \downarrow}$, and

$$
V_{\mathbf{q}}^{\uparrow \uparrow}=V_{\mathbf{q}}^{\downarrow \downarrow}=V_{\mathbf{q}}^{\uparrow \downarrow}=V_{\mathbf{q}}^{\downarrow \uparrow}
$$

In Fig.(2),$V_{x x}(\mathbf{q})$ and $V_{z z}(\mathbf{q})$ is shown for two different areal densities. At large areal density $V_{z z}(\mathbf{q})$ is negligible compared with $V_{x x}(\mathbf{q})$ and the corresponding Hamiltonian is effectively the DQW Hamiltonian. The non-zero isopotential matrix elements in this case are $V_{00}(\mathbf{q})$ and $V_{x x}(\mathbf{q})$ and may be obtained by applying Eq.(17) in Eq.(11). These matrix elements have been exploited before in the study of double quantum wells [7,:8] where the SWQW is effectively a DQW. Note that in this case $V_{0 z}(\mathbf{q})$ is nearly zero and the effect of many body exchange part of Hamiltonian on $\Delta_{S A S}$ is negligible so that, $\Delta_{S A S}=\Delta_{S A S}^{0}$. In the next section we will see that this is true even in the presence of strong magnetic field. Therefore in the DQW or high density SWQW problems, the effect of the many body part of Hamiltonian on $\Delta_{S A S}$ is zero.

Therfore one may decompose the electron-electron interaction Hamiltonian Eq.(9) into three terms

$$
\hat{V}=\hat{V}_{D L}+\hat{V}_{B}+\hat{V}_{U} .
$$

where $\hat{V}_{D L}, \hat{V}_{B}$ and $\hat{V}_{U}$ are the double layer, balanced and unbalanced part of electronelectron interaction respectively

$$
\hat{V}_{D L}=\frac{1}{2} \int \frac{d^{2} \mathbf{q}}{(2 \pi)^{2}}\left\{V_{0}(\mathbf{q}) \hat{\rho}(-\mathbf{q}) \hat{\rho}(\mathbf{q})+V_{x}(\mathbf{q}) \hat{\sigma}^{x}(-\mathbf{q}) \hat{\sigma}^{x}(\mathbf{q})\right\}
$$


$\hat{V}_{B}=\frac{1}{2} \int \frac{d^{2} \mathbf{q}}{(2 \pi)^{2}}\left\{V_{0 z}(\mathbf{q})\left[\hat{\rho}(-\mathbf{q}) \hat{\sigma}^{z}(\mathbf{q})+\hat{\sigma}^{z}(-\mathbf{q}) \hat{\rho}(\mathbf{q})\right]+V_{z}(\mathbf{q}) \hat{\sigma}^{z}(-\mathbf{q}) \hat{\sigma}^{z}(\mathbf{q})\right\}$
$\hat{V}_{U}=\frac{1}{2} \int \frac{d^{2} \mathbf{q}}{(2 \pi)^{2}}\left\{V_{0 x}(\mathbf{q})\left[\hat{\rho}(-\mathbf{q}) \hat{\sigma}^{x}(\mathbf{q})+\hat{\rho}(-\mathbf{q}) \hat{\sigma}^{x}(\mathbf{q})\right]+V_{x z}(\mathbf{q})\left[\hat{\sigma}^{x}(-\mathbf{q}) \hat{\sigma}^{z}(\mathbf{q})+\hat{\sigma}^{z}(-\mathbf{q}) \hat{\sigma}^{x}(\mathbf{q})\right]\right\}$.

$\hat{V}_{D L}$ has the same form as in a DQW problem. The last two terms are corrections to the DQW problem, due to non-zero electron density in the middle of the SWQW. These two terms are negligible when the density of electrons in the middle of the SWQW is small enough.

\section{MANY BODY HAMILTONIAN OF SWQWS IN PRESENCE OF STRONG PERPENDICULAR MAGNETIC FIELD}

In this section we study the SWQW in the presence of strong magnetic field where all electrons are located in the LLL. We exploit the techniques of projection onto the LLL which has been developed by Girvin and Jach [9]. We will show that this technique is equivalant with the Hartree-Fock approximation at filling factor $\nu=1$.

\section{A. Projection onto the lowest Landau level}

Taking the perpendicular component of the magnetic field to be strong, we restrict the Hilbert space to the lowest Landau level (LLL) and exploit the LLL projection formalism which was developed to study of collective excitations in the FQHE [9,10]. The interaction part of the Hamiltonian which describes the low-energy excitations of the system obtained in Eqs.(18) through (21), may be projected onto the LLL by using

$$
\begin{gathered}
\overline{\rho_{-\mathbf{q}} \rho_{\mathbf{q}}}=\bar{\rho}_{-\mathbf{q}} \bar{\rho}_{\mathbf{q}}-\bar{\rho}_{\mathbf{q}=0} e^{-q^{2} / 2} \\
\overline{\sigma_{-\mathbf{q}}^{a} \sigma_{\mathbf{q}}^{b}}=\bar{\sigma}_{-\mathbf{q}}^{a} \bar{\sigma}_{\mathbf{q}}^{b}-\left(i \epsilon^{a b c} \bar{\sigma}_{\mathbf{q}=0}^{c}+\delta^{a b} \bar{\rho}_{\mathbf{q}=0}\right) e^{-q^{2} / 2} \\
\overline{\sigma_{-\mathbf{q}}^{\mu} \rho_{\mathbf{q}}}=\bar{\sigma}_{-\mathbf{q}}^{\mu} \bar{\rho}_{\mathbf{q}}-\bar{\sigma}_{\mathbf{q}=0}^{\mu} e^{-q^{2} / 2}
\end{gathered}
$$


In the above equations $\bar{\rho}_{q}$ and $\bar{\sigma}_{q}^{\mu}$ are the projected total charge density and $\mu$ th component of isospin density operators respectively 12

$$
\begin{gathered}
\bar{\rho}(\mathbf{q})=\frac{1}{\sqrt{A}} \sum_{j=1}^{N} \overline{e^{-i \mathbf{q} \cdot \mathbf{r}_{j}}}=\frac{1}{\sqrt{A}} \sum_{j=1}^{N} e^{-\frac{|q|^{2}}{4}} \tau_{q}(j) \\
\overline{\sigma^{\mu}}(\mathbf{q})=\frac{1}{\sqrt{A}} \sum_{j=1}^{N} \overline{e^{-i \mathbf{q} \cdot \mathbf{r}_{j}}} \sigma_{j}^{\mu}=\frac{1}{\sqrt{A}} \sum_{j=1}^{N} e^{-\frac{|q|^{2}}{4}} \tau_{q}(j) \sigma_{j}^{\mu},
\end{gathered}
$$

where $q=\ell_{0}\left(q_{x}+i q_{y}\right)$. The magnetic translation operator for the $j$ th particle

$$
\tau_{q}(j)=e^{-i q \frac{\partial}{\partial z_{j}}-\frac{i}{2} q^{*} z_{j}}
$$

is a unitary operator satisfying the closed Lie algebra

$$
\begin{gathered}
\tau_{q} \tau_{k}=\tau_{q+k} e^{\frac{i}{2} q \wedge k} \\
{\left[\tau_{q}, \tau_{k}\right]=2 i \tau_{q+k} \sin \frac{q \wedge k}{2},}
\end{gathered}
$$

where $q \wedge k \equiv \ell_{0}^{2}(\mathbf{q} \times \mathbf{k}) \cdot \hat{\mathbf{z}}$.

One may find the commutation relations between isospin density operators by using Eq. (25)

$$
\begin{gathered}
{\left[\bar{\sigma}_{k_{1}}^{0}, \bar{\sigma}_{k_{2}}^{\mu}\right]=\left(e^{k_{1} k_{2}^{*} / 2}-e^{k_{2} k_{1}^{*} / 2}\right) \bar{\sigma}_{k_{1}+k_{2}}^{\mu},} \\
{\left[\bar{\sigma}_{k_{1}}^{a}, \bar{\sigma}_{k_{2}}^{b}\right]=\left(e^{k_{1} k_{2}^{*} / 2}-e^{k_{2} k_{1}^{*} / 2}\right) \bar{\sigma}_{k_{1}+k_{2}}^{0} \delta^{a b}+\left(e^{k_{1} k_{2}^{*} / 2}+e^{k_{2} k_{1}^{*} / 2}\right) i \epsilon^{a b c} \bar{\sigma}_{k_{1}+k_{2}}^{c},}
\end{gathered}
$$

where $k^{*}$ is the complex conjugate of $k$. Note that 4-components are labeled by Greek indices and Latin indices denotes spatial components. The tunneling term is renormalized by the last linear term of Eq.(22). The projected tunneling term is thus

$$
\bar{T}=-\frac{\Delta_{S A S}^{B}}{2} \bar{\sigma}^{z}(\mathbf{q}=0)
$$

where $\Delta_{S A S}^{B}$ is the renormalized energy difference between up and down isospin states. Due to the effect of the strong magnetic field on the exchange energy, we have 


$$
\Delta_{S A S}^{B}=\Delta_{S A S}+2 \int \frac{d^{2} \mathbf{q}}{(2 \pi)^{2}} V_{0 z}(\mathbf{q}) e^{-q^{2} \ell_{0}^{2} / 2} .
$$

One may obtain the analytical expression for renormilized tunneling, Eq.(28), using the Hartree-Fock calculation where the many body ground state wave function may be written as a single Slater determinant. A particular class of single Slater deteminant at $\nu=1$ in the Landaue guage which yields to Eq.(28) can be written in the form [12]

$$
|\Psi\rangle=\prod_{X}\left(\hat{C}_{X \uparrow}^{\dagger} \cos \frac{\theta(X)}{2}+\hat{C}_{X \downarrow}^{\dagger} \sin \frac{\theta(X)}{2} e^{i \varphi(X)}\right)|0\rangle
$$

where $|0\rangle$ is fermionic vacuum and $\hat{C}_{X \uparrow, \downarrow}^{\dagger}$ creates an electron in the Symmetric (Antiymmetric) or Left (Right) in orbit $\phi_{X}$, respectively. Taking the many body wave function Eq.(29) and using the HFA, leads to Eq.(28). We may use Eq.(28) for any fractional filling factors e.g. $\nu=1 / 2$ to evaluate the renormalized tunneling. Note that this generalization is just a simple extrapolation of Eq.(28) to fractional filling factors. As one may see from Eq.(28), for a given well width and areal density, $V_{0 z}(\mathbf{q})$ is given and $\Delta_{S A S}^{B}$ is just a monotonic decreasing function of magnetic field

$$
\Delta_{S A S}^{B}(\nu)-\Delta_{S A S} \propto \mp \nu^{-1 / 2},
$$

where $\nu$ is the Landau level filling factor. The sign is specified by the integral in Eq.(28), which depends on the electron areal density. Our numerical calculation shows that in the domain of experimental densities, the sign is minues, i.e. the renormalized $\Delta_{S A S}$ is less than the bare one. It is instructive to write $\Delta_{S A S}^{B}(\nu)$ in terms of the renormalized $\Delta_{S A S}$ at given filling factor i.e. $\nu=1$ and the bare $\Delta_{S A S}$

$$
\Delta_{S A S}^{B}(\nu)=\left(1-\nu^{-1 / 2}\right) \Delta_{S A S}+\nu^{-1 / 2} \Delta_{S A S}^{B}(\nu=1) .
$$

We may use Eq.(31) in order to find $\Delta_{S A S}^{B}(\nu=1 / 2)$. To the best of our knowledge there is no reliable measurement or calculation to find $\Delta^{B} S A S$ and interlayer seperation $(d)$ at fractional filling factor. Our numerical results for realistic samples has been shown in Table I. According to thses results, we may conclude that the renormalized tunneling term at 
$\nu=1 / 2$ is large enough that 331 Halperin's wave function, which is exact in the limit of vanishing tunneling, is not the best variational wavefunction to describe the ground state. However, it is not obvious that $\Delta_{S A S}^{B}(\nu \neq 1)$ is the relavant parameter to the FQH gap corresponding to the FQHE at $\nu \neq 1$.

At the end of this section and for further calculations we list the projected isopotentials onto LLL

$$
\begin{gathered}
\bar{V}=\bar{V}_{D L}+\bar{V}_{B}+\bar{V}_{U} \\
\bar{V}_{D L}=\frac{1}{2} \int \frac{d^{2} \mathbf{q}}{(2 \pi)^{2}}\left\{V_{0}(\mathbf{q}) \bar{\rho}(-\mathbf{q}) \bar{\rho}(\mathbf{q})+V_{x}(\mathbf{q}) \bar{\sigma}^{x}(-\mathbf{q}) \bar{\sigma}^{x}(\mathbf{q})\right\} \\
\bar{V}_{B}=\frac{1}{2} \int \frac{d^{2} \mathbf{q}}{(2 \pi)^{2}}\left\{V_{0 z}(\mathbf{q})\left[\bar{\rho}(-\mathbf{q}) \bar{\sigma}^{z}(\mathbf{q})+\bar{\sigma}^{z}(-\mathbf{q}) \bar{\rho}(\mathbf{q})\right]+V_{z}(\mathbf{q}) \bar{\sigma}^{z}(-\mathbf{q}) \bar{\sigma}^{z}(\mathbf{q})\right\} \\
\bar{V}_{U}=\frac{1}{2} \int \frac{d^{2} \mathbf{q}}{(2 \pi)^{2}}\left\{V_{0 x}(\mathbf{q})\left[\bar{\rho}(-\mathbf{q}) \bar{\sigma}^{x}(\mathbf{q})+\bar{\sigma}^{x}(-\mathbf{q})\right] \bar{\rho}(\mathbf{q})+V_{x z}(\mathbf{q})\left[\bar{\sigma}^{x}(-\mathbf{q}) \bar{\sigma}^{z}(\mathbf{q})+\bar{\sigma}^{z}(-\mathbf{q}) \bar{\sigma}^{x}(\mathbf{q})\right]\right\}
\end{gathered}
$$

As we mentioned in the zero magnetic field case, the last two terms of Eq.(32), are negligible when the density of electrons in the middle of SWQWs is small enough, and the system is effectively a DQW with renormalized $\Delta_{S A S}^{B}$ (which is nearly equal to the bare one) and hence one may expect that the many body quantum Hall ground state is described by the Halperin variational wave functions as it is in DQWs.

\section{LOCAL DENSITY FUNCTIONAL RESULTS FOR SWQWS IN STRONG MAGNETIC FIELD}

The system we study numerically in this section is a wide, single, GaAs quantum well in the presence of a strong perpendicular magnetic field. We consider a slab of GaAs of thickness $W$ confined between two infinitely high barriers, which represent $\mathrm{Al}_{\mathrm{x}} \mathrm{Ga}_{1-\mathrm{x}} \mathrm{As}$. This system is doped by Si delta layers, separated symmetrically, by distance $\mathrm{h}$ from the 
GaAs and which have ionized donor concentration $N_{s}$ per unit area. We apply the local density functional approximation (LDFA) to find eigenvalues and eigenfunctions of the many body system. By this technique one may find the charge distribution function, eigenenergies and the effective potential, which are calculated by self-consistent Kohn-Sham equations in a strong magnetic field, i.e. by solving the Poisson and Schrödinger equations simultaneously. In the presence of a strong magnetic field, we restrict our attention to the lowest Landau level (LLL), for which the single-body wavefunctions, in symmetric gauge and polar coordinates are

$$
\varphi_{m}(r, \theta)=\frac{1}{\sqrt{2 \pi \ell_{0}^{2} 2^{m} m !}}\left(\frac{r}{\ell_{0}}\right)^{m} \exp \left(\frac{-r^{2}}{4 \ell_{0}^{2}}\right) e^{i m \theta}
$$

The full one-body wavefunction corresponding to $j$ th subband may be written

$$
\Phi_{j, m}(r, \theta, z)=\varphi_{m}(r, \theta) \psi_{j}(z)
$$

with the corresponding charge distribution function

$$
n(\mathbf{x})=\sum_{j} \sum_{m}\left|\varphi_{m}(r, \theta)\right|^{2}\left|\psi_{j}(z)\right|^{2}
$$

In the strong magnetic field regime, where all electrons are accomodated within the LLL and execute cyclotron orbits with a common kinetic energy, the summation over $m$ yields $\frac{1}{2 \pi \ell_{0}^{2}}$ independent of $r$, the in-plain position of electrons. In the LLL where the filling factor is a fractional number between zero and one, only the first subband is filled, and the ground state can be described as an isospin ferromagnet, i.e., a phase coherent state [11]. In zero magnetic field under typical experimental circumstances [1,2], the second subband is partially filled depending on areal density. In a sufficiently strong magnetic field, the Landau level degeneracy is high enough that electrons which were in second subband in zero magnetic field, will be located in the first subband. The density distribution function in Eq.(38) then reduces to

$$
n(z)=\frac{\nu}{2 \pi \ell_{0}^{2}}\left|\psi_{0}(z)\right|^{2} .
$$


Following a procedure similar to that used for $\mathrm{GaAs} / \mathrm{Al}_{\mathrm{x}} \mathrm{Ga}_{1-\mathrm{x}} \mathrm{As}$ heterojunctions at zero magnetic field [18], we solve for the quantized energy level of $j$ th subband, $E_{j}$ and its corresponding envelope function $\psi_{j}(z)$ satisfing the following Schrödinger equation

$$
\left[\frac{-\hbar^{2}}{2 m^{*}} \frac{d^{2}}{d z^{2}}+V_{\mathrm{eff}}(z)\right] \psi_{j}(z)=E_{j} \psi_{j}(z),
$$

Here $m^{*}$ is the electron effective mass of GaAs and $V_{\text {eff }}(z)$ is the one electron effective potential of Kohn-Sham local density functional theory (LDFT) which splits into three different contributions

$$
V_{\mathrm{eff}}(z)=V_{b}(z)+V_{h}(z)+V_{x c}^{\uparrow \uparrow}(z) .
$$

The quantity $V_{b}(z)$ is the built in potential due to the infinite barrier and $V_{h}(z)$ is the Hartree term due to all coulomb interactions between electrons in the presence of a uniform density of background ions

$$
V_{h}(z)=-\frac{2 \pi e^{2}}{\epsilon} \int d z^{\prime}\left|z-z^{\prime}\right| n\left(z^{\prime}\right) .
$$

$V_{x c}^{\uparrow \uparrow}(z)$ is the exchange-correlation potential in the Kohn-Sham local density functional approximation. The LDFA exchange-correlation energy, which has been studied by Vignale and Rasolt [19], is a functional of the scalar charge density and vector current density. In our approximation, we ignore the current density term in the exchange-correlation energy and we assume that all electrons are spin polarized along the magnetic field. The best functional for our purposes is the Vosko-Wilk-Nusair exchange-correlation energy, $V_{x c}^{\uparrow \uparrow}(z)$, which is parametrising the Ceperly-Alder Monte Carlo calculation [21], and which has been applied to three dimensional itinerant ferromagnets [20].

Results of our numerical calculations for $\Delta_{S A S}$ for $\nu=1$ filling factor and several $N_{s}$ are listed in Table I. In this Table the parameter d, which we define as the distance between the peaks in the charge-density profile as shown in Fig.(5), decreases as $N_{s}$ is lowered. The values of $N_{s}$ which we used have been taken from Suen et al.'s experimental data [2]. At this point, we may compare the results of the strong magnetic field LDFA and calculation 
of the renormalized $\Delta_{S A S}^{B}$ of the many body Hamiltonian Eq. (28), in which the tunneling term is enhanced by electron-electron interactions. We will evaluate the integral in Eq.(28) by knowing the $V_{0 z}(\mathbf{q})$ which has been defined in Eq.(11). We use the zero magnetic field orthonormal wave functions of the first and second subband to find the $V_{0 z}(\mathbf{q})$ and hence $\Delta_{S A S}^{B}$.

We used the self-consistent LDFA symmetric-antisymmetric wave functions for zero magnetic field to calculate $\Delta_{S A S}^{B}$ numerically. The form of exchange-correlation potential which we chose is Hedin and Lundqvist [22] which has been used in an investigation of $\mathrm{GaAs} / \mathrm{Al}_{\mathrm{x}} \mathrm{Ga}_{1-\mathrm{x}} \mathrm{As}$ hetero junctions in zero magnetic field [18].

One may define the relative difference between the bare and renormalized tunneling term, $\delta=\frac{\Delta_{S A S}-\Delta_{S A S}^{B}}{\Delta_{S A S}}$ which is a quantity to measure the deviation of SWQW's charge distribution due to the strong magnetic field. $\delta$ may calculated by the integral of $V_{0 z}(\mathbf{q}) e^{-q^{2} / 2}$ which is shown for different densities in Fig.(33). The result of integration is positive (negative) at high (low) densities and depends on the long wavelength oscillations of $V_{0 z}(\mathbf{q})$. Our numerical calculation shows that $\delta$ is small and even negative at low densities and becomes positive with increasing the density. For example, at $N_{s}=0.5 \times 10^{11} \mathrm{~cm}^{-2}$, we find $\delta=-0.1$ which is relatively small due to the long wavelength cancellation of $V_{0 z}(\mathbf{q})$ upon integration, and therefore the electron density $n(z)$ is not affected enormously by the strong magnetic field. Note that the sign of $\delta$ is negative, which means that the tunneling term is increased by the strong magnetic field. In Fig.(田), $V_{0 z}(\mathbf{q})$ is plotted for $N_{s}=0.5 \times 10^{11} \mathrm{~cm}^{-2}$ and $W=680(\AA)$. In Fig.(河) we compare the electron density of a SWQW in zero and strong magnetic field by LDFA calculations. This comparison for $N_{s}=0.5 \times 10^{11} \mathrm{~cm}^{-2}$ and $W=680(\AA)$ shows that the two densities are very close which is indicates qualitative agreement between the LDFA and many body calculations.

At very large densities $V_{0 z}(\mathbf{q})$ is a smoothly negative but close to zero and hence $\delta$ is an small negative number. It is important to note that $\delta$ is very small in the DQW regime which is equivalant to large SWQW densities and is strictly zero for the true DQW case. In the domain of densities which is of interest for us, both calculation of LDFA and the many 
body Hamiltonian show a decrease of $\Delta_{S A S}$ due to the strong magnetic field on the order of 1 degree of Kelvin. We conclude that the effect of strong magnetic field on the electron density $n(z)$ is negligible at very low and very high areal electron density. At intermediate densities, the effect of the strong magnetic field is significant and the electron distribution along the $\hat{z}$-direction is changed. One ought to be able to measure this effect experimentally.

In Fig.(5) we plot the result of a self-consistent LDFA calculation at strong magnetic field for $N_{s}=3.1 \times 10^{11} \mathrm{~cm}^{-2}$ where the difference of charge density in zero magetic field and strong magnetic field is relatively large. In strong magnetic field, the charge density is proportional to square of the lowest subband wave function. Therefore the result of nonzero parameter $d$ is due to tunneling effect through $V_{\text {eff }}(z)$ barrier where the eigenenergy is less than the top of effective potential. Obviously in the presence of strong perpendicular magnetic field, $d$ is a monotonicaly decreasing function of $N_{s}$, and at a critical density $N_{s c}$ and critical width $W_{c}$, the parameter $d$ continously goes to zero. For example our numerical calculation shows this transition between two-layer and one-layer quantum wells occurs at $N_{s} \equiv N_{s c}=0.5 \times 10^{11} \mathrm{~cm}^{-2}$ for a SWQW's width of $680(\AA)$. To the best of our knowledge, there is no exprimental results to report the value of $\Delta_{S A S}^{B}$ and $d$ [25] to check with our numerical results.

\section{COLLECTIVE MODES EXCITATIONS OF SWQWS FRACTIONAL QUANTUM HALL STATE}

Recently, there has been much interest in experimental work in DQWs and SWQWs [1, 3], a remarkable effect is observed, namely the absence of certain IQHE at odd $\nu$ for sufficiently small $\Delta_{S A S}$ and large interalayer distance $(d)$. These experimental observations have been explained in a phase diagram proposed by MacDonald et al. [7] for the presence or absence of the IQHE for different DQW parameters, associated with the loss of the isomagnon excitation gap. We generalize their model to SWQWs with the help of experimental parameters used by Suen et al. [2]. In the previous sections we found that in the presence of strong prependicular 
magnetic field only the first subband is occupied where all electrons are in the isospin state, up. One may expect that the low lying excitations are isospin waves (isomagnons) corresponding to a single flipped isospin which propagates through the system. Therefore we may describe the isomagnons as a bound state of an electron of one isospin with a hole of opposite isospin. The Hamiltonian describing the low-energy excitation of the SWQW system has been obtained in the preceding sections

$$
H=-\frac{\Delta_{S A S}^{B}}{2} \sigma_{z}(\mathbf{q}=0)+\frac{1}{2} \int \frac{d^{2} \mathbf{q}}{(2 \pi)^{2}} \bar{\sigma}_{\mu}(-\mathbf{q}) V^{\mu \nu}(\mathbf{q}) \bar{\sigma}_{\nu}(\mathbf{q})
$$

where the isopotentials $V^{\mu \nu}(\mathbf{q})$ are defined by Eq.(11). The calculation of the isomagnon collective-mode energy associated with the first to second subband excitation, which is a type of isospin wave in isospin space, is based on the single mode approximation [7] and the free boson model of Holstein-Primakoff transformation which has been exploited in the study of ferromagnetic spin waves problem [13]. The normalized isomagnon wave function is given by

$$
\left|\Psi_{-}(\mathbf{k})\right\rangle=\frac{e^{\ell_{0}^{2} k^{2} / 4}}{\sqrt{N}} \bar{S}_{-}(\mathbf{k})\left|\Psi_{0}\right\rangle .
$$

Here $\left|\Psi_{0}\right\rangle=\bigotimes_{i}|\uparrow\rangle_{i}$ is the fully isospin polarized ground state of the system in which all electrons are located within the first subband and $\bar{S}_{ \pm}(\mathbf{k})=\left(\bar{\sigma}_{x}(\mathbf{k}) \pm i \bar{\sigma}_{y}(\mathbf{k})\right) / 2$ are isospin lowering (raising) operators. In the absence of an external perpendicular electeric field where the system is balanced and the first (second) subbands are symmetric (antisymmetric) respectively, the full Hamiltonian can be separated into terms which conserves $S_{z}^{t o t}\left(H_{0}\right)$

$$
H_{0}=T+V_{0}+H^{\prime}
$$

where $T$ is the tunneling term and

$$
\begin{aligned}
& V_{0}=\frac{1}{2} \int \frac{d^{2} \mathbf{q}}{(2 \pi)^{2}}\left(V_{0}(\mathbf{q}) \bar{\rho}(-\mathbf{q}) \bar{\rho}(\mathbf{q})+V_{z}(\mathbf{q}) \bar{\sigma}^{z}(-\mathbf{q}) \bar{\sigma}^{z}(\mathbf{q})+\right. \\
& \left.V_{0 z}(\mathbf{q})\left[\bar{\sigma}^{z}(-\mathbf{q}) \bar{\rho}(\mathbf{q})+\bar{\rho}(-\mathbf{q}) \bar{\sigma}^{z}(\mathbf{q})\right]\right),
\end{aligned}
$$

and a term $H^{\prime}$ which creates (annihilates) a pair of isomagnons and hence changes $S_{z}^{\text {tot }}$ by 2: 


$$
H^{\prime}=\frac{1}{2} \int \frac{d^{2} \mathbf{q}}{(2 \pi)^{2}} V_{x}(\mathbf{q})\left(\bar{S}_{+}(-\mathbf{q}) \bar{S}_{+}(\mathbf{q})+\bar{S}_{-}(-\mathbf{q}) \bar{S}_{-}(\mathbf{q})\right)
$$

Using the single mode approximation [14] and commutation relation Eq.(26) yields

$$
\left\langle\Psi_{-}(\mathbf{k})\left|H_{0}\right| \Psi_{-}(\mathbf{k})\right\rangle=E_{0}+\varepsilon(\mathbf{k})
$$

Here $E_{0}=\left\langle\Psi_{0}\left|H_{0}\right| \Psi_{0}\right\rangle$ and

$$
\begin{array}{r}
\varepsilon(\mathbf{k})=\Delta_{S A S}^{B}+\frac{\nu}{2 \pi \ell_{0}^{2}} V_{x}(\mathbf{k}) e^{-\ell_{0}^{2} k^{2} / 2}-\int \frac{d^{2} \mathbf{q} e^{-\ell_{0}^{2} q^{2} / 2}}{(2 \pi)^{2}} V_{0 z}(\mathbf{q})(\tilde{h}(\mathbf{q})+1) \\
+\int \frac{d^{2} \mathbf{q} e^{-\ell_{0}^{2} q^{2} / 2}}{(2 \pi)^{2}}\left(V_{x}(\mathbf{q}) \tilde{h}(\mathbf{q}+\mathbf{k})-\left[V_{0}(\mathbf{q})+V_{z}(\mathbf{q})\right] \tilde{h}(\mathbf{q})\right) \\
+\int \frac{d^{2} \mathbf{q} e^{-\ell_{0}^{2} q^{2} / 2}}{(2 \pi)^{2}}\left(\left[V_{0}(\mathbf{q})+V_{z}(\mathbf{q})\right] \tilde{h}(\mathbf{q}) e^{i \ell_{0}^{2} \mathbf{q} \cdot(\hat{z} \times \mathbf{k})}\right)
\end{array}
$$

where $\tilde{h}(\mathbf{q})=h(\mathbf{q}) \exp \left(\ell_{0}^{2} q^{2} / 2\right)$ and $h(\mathbf{q})$ is the Fourier transform of the pair-correlation function. For $\nu=1$ this is given by $h(\mathbf{q})=-\exp \left(-\ell_{0}^{2} q^{2} / 2\right)$. These results generalize the results which were obtained previously [7] for the special case $V_{z}(\mathbf{q})=V_{0 z}(\mathbf{q}) \equiv 0$ for DQWs. The physical interpretation of Eq. (49) has been explained in the study of DQW system by MacDonald et al. [7]. The first term of Eq.(49) is the dressed excitation energy from the first to the second subband, the second term is the Hartree correction due to the isospin dependent part of the electron-electron interaction and the third term is the Hartree enhancement of $\Delta_{S A S}^{B}$ associated with isospin coulomb interaction and is zero for $\nu=1$. The fourth term is the difference between the self-energy of electrons in the second and first subbands and the last term is the energy associated with the electron-hole interaction.

The full Hamiltonian Eq.(43) may be approximated by a soluble quadratic bosonic effective Hamiltonian by application of the Holstein-Primakoff transformation

$$
H_{b}=E_{0}+\sum_{\mathbf{k}}\left(\varepsilon(\mathbf{k}) \hat{b}_{\mathbf{k}}^{\dagger} \hat{b}_{\mathbf{k}}+\frac{1}{2} \lambda(\mathbf{k})\left(\hat{b}_{\mathbf{k}} \hat{b}_{-\mathbf{k}}+\hat{b}_{\mathbf{k}}^{\dagger} \hat{b}_{-\mathbf{k}}^{\dagger}\right)\right),
$$

where $\hat{b}_{\mathbf{k}}^{\dagger}\left(\hat{b}_{\mathbf{k}}\right)$ are bosonic creation (annihilation) operators. Here $H_{0}$ and $H^{\prime}$ are transformed to quadratic boson pieces $\sum_{\mathbf{k}} \varepsilon(\mathbf{k}) \hat{b}_{\mathbf{k}}^{\dagger} \hat{b}_{\mathbf{k}}$ and $\frac{1}{2} \sum_{\mathbf{k}} \lambda(\mathbf{k})\left(\hat{b}_{\mathbf{k}} \hat{b}_{-\mathbf{k}}+\hat{b}_{\mathbf{k}}^{\dagger} \hat{b}_{-\mathbf{k}}^{\dagger}\right)$. The latter creates or destroys an isomagnon pair with opposite momentum and is responsible for broken global gauge symmetry. 
One may obtain $\lambda(\mathbf{k})$ by fitting to the exact expression for the matrix element of $H^{\prime}$ between the zero and two isomagnon states, which leads to

$$
\lambda(\mathbf{k})=\frac{\nu}{2 \pi \ell_{0}^{2}} V_{x}(\mathbf{k}) e^{-\ell_{0}^{2} k^{2} / 2}+\int \frac{d^{2} \mathbf{q} e^{-\ell_{0}^{2} q^{2} / 2}}{(2 \pi)^{2}} V_{x}(\mathbf{q}) \tilde{h}(\mathbf{q}+\mathbf{k}) e^{i \ell_{0}^{2} \mathbf{q} \cdot(\tilde{z} \times \mathbf{k})} .
$$

The effective Hamiltonian $H_{b}$ may be diagonalized by Bogoliubov transformation and the resulting isospin-excitation energies are given by

$$
E(\mathbf{k})=\sqrt{\varepsilon^{2}(\mathbf{k})-\lambda^{2}(\mathbf{k})} .
$$

The energy of the system is reduced by generating virtual isomagnon-pairs and therefore, pairing of isomagnons is prefered for the ground state. At $\nu=1$ the dispersion relation Eq.(52) has a gap at $\mathbf{k}=0$ corresponding to $\Delta_{S A S}^{B}$ where the quantum Hall gap is specified by the local minimum of Eq.(52). For zero tunneling, Eq.(52) describes a gapless goldestone mode corresponding to spontaneous global U(1) symmerty breaking in the ground state describing a superfluid associated with fluctuation in $S_{z}^{\text {tot }}$ [23], in spite of violating the U(1) symmetry of the original Hamiltonian Eq.(43) where the symmetry group of SWQWs Hamiltonian is $Z_{2}$ corresponding to $\sigma^{y} \rightarrow-\sigma^{y}$, while the DQWs Hamiltonian has $\mathrm{U}(1)$ symmetry group when $V_{z}(\mathbf{q})=V_{0 z}(\mathbf{q})=0$. Note that $Z_{2}$ symmetry of Hamiltonian is also broken in the presence of the tilted magnetic field as we mentioned before. However at $\nu \neq 1$ where the third term of Eq.(49) is nonzero but constant (depending on total filling factor), the collective modes are gapful even at zero tunneling term and there is no goldestone mode corresponding to spontaneous symmetry breaking while in DQW, spontaneous global $\mathrm{U}(1)$ symmetry breaking occurs at any filling factor $\nu$, generates the goldestone modes for zero tunneling. The collective modes are understood by the pole of isospin density-density response function within LLL which has been called isomagnons. In our approximation it corresponds to the local minimum of dispersion curve Fig.(6), which are gapful or gapless in special circumstances as we mentioned above. Note that this low-energy mode is the analogous to those in the Feynman theory of superfluid ${ }^{4} \mathrm{He}[15]$. One may associate the odd total filling factor quantum Hall effect with isomagnons gap where $\Delta_{S A S}^{B}$, the energy 
difference between the subbands is the the relevant energy scale to compare with the inplane coulomb energy $e^{2} /\left(\epsilon_{0} \ell_{0}\right)$, i.e. the competition between $\Delta_{S A S}^{B}$ and the coulomb energy yields the correspondig QHE. Therefore the phase boundary between incompressible and compressible states can be defined by the collapse of the gap which occurs at certain $\Delta_{S A S}^{B}$ and $d$, while the corresponding gap at even total filling factors has been identified by the charge excitations (magnetorotons) which is infinitely large in our approximation [15]. One may generalize the above formalism for FQHE associated with a many body energy gap due to correlation between electrons in different layers which is destroyed if they are uncorrelated by increasing the layer distance.

At this point we may study the effect of applying a perpendicular electric field on the dispersion relation of collective modes by considering an imbalanced SWQW. As we disscused above, inversion symmetry is broken by perpendicular electric field and the first (second) subbands are no longer symmetric (antisymmetric). There is also an additional term in collective mode Hamiltonian, Eq.(45-47), due to the external electric field Hamiltonian Eq.(21). We have to mention that, the corresponding additional term, $V_{U}$, changes $S_{z}^{\text {tot }}$ by 1 . In this case the charge excitations couples with isomagnons and the ground state may be described by the virtual isomagnon-plasmon pairs. One may divide $V_{U}$ into two parts, $V_{U}=V_{U}^{0}+V_{U}^{z}$, where $V_{U}^{0}\left(V_{U}^{z}\right)$ can also be separated into two parts

$$
\begin{aligned}
& V_{U}^{0}=V_{U}^{+(0)}+V_{U}^{-(0)}, \\
& V_{U}^{z}=V_{U}^{+(z)}+V_{U}^{-(z)},
\end{aligned}
$$

where we define $V_{U}^{ \pm}$by

$$
\begin{gathered}
V_{U}^{ \pm(0)}=\frac{1}{2} \int \frac{d^{2} \mathbf{q}}{(2 \pi)^{2}} V_{0 x}(\mathbf{q})\left[\bar{\rho}(-\mathbf{q}) \bar{S}_{ \pm}(\mathbf{q})+\bar{S}_{ \pm}(-\mathbf{q}) \bar{\rho}(\mathbf{q})\right] \\
V_{U}^{ \pm(z)}=\frac{1}{2} \int \frac{d^{2} \mathbf{q}}{(2 \pi)^{2}} V_{x z}(\mathbf{q})\left[\overline{S^{z}}(-\mathbf{q}) \bar{S}_{ \pm}(\mathbf{q})+\bar{S}_{ \pm}(-\mathbf{q}) \overline{S^{z}}(\mathbf{q})\right]
\end{gathered}
$$

Again $V_{U}^{0}\left(V_{U}^{z}\right)$ will be bosonized by the Holestein-Primakoff transformation 


$$
\begin{gathered}
H_{b}^{0}=\frac{1}{2} \sum_{\mathbf{k}} \eta(\mathbf{k})\left(\hat{a}_{\mathbf{k}} \hat{b}_{-\mathbf{k}}+\hat{b}_{-\mathbf{k}}^{\dagger} \hat{a}_{\mathbf{k}}^{\dagger}\right), \\
H_{b}^{z}=\frac{1}{2} \sum_{\mathbf{k}} \zeta(\mathbf{k})\left(\left(\hat{n}_{\mathbf{k}}-\frac{1}{2}\right) \hat{b}_{-\mathbf{k}}+\hat{b}_{-\mathbf{k}}^{\dagger}\left(\hat{n}_{\mathbf{k}}-\frac{1}{2}\right)\right),
\end{gathered}
$$

where we have used the bosonic hard sphere condition [17] for Eq.(55b). Here $\hat{a}_{\mathbf{k}}^{\dagger}\left(\hat{a}_{\mathbf{k}}\right)$ is the charge density excitation creation (annihilation) operator, which yield no pole in the densitydensity response function within LLL [8], e.g. magnetorotons with a huge gap equivalent to $\hbar \omega_{c}$ and $\hat{n}_{\mathbf{k}}$ which is bosonic number operator. Therefore, Eq.(55) may describe coupling between isomagnons and magnetorotons due to external electric field bias.

Following the same calculation we have done for the ballanced SWQW Eq.(51), we may determine $\eta(\mathbf{k})(\zeta(\mathbf{k}))$ from the exact result for matrix element of $V_{U}^{0}\left(V_{U}^{z}\right)$ between the zero and two isomagnon-magnetoroton states

$$
\begin{array}{r}
\eta(\mathbf{k})=\int \frac{d^{2} \mathbf{q} e^{-\ell_{0}^{2} q^{2} / 2}}{(2 \pi)^{2}} V_{0 x}(\mathbf{q})\left(1+\tilde{h}(\mathbf{q})+\tilde{h}(\mathbf{k})+e^{i \ell_{0}^{2} \mathbf{q} \cdot(\hat{z} \times \mathbf{k})} \tilde{h}(\mathbf{k}+\mathbf{q})\right. \\
\left.+\frac{e^{\left(q \bar{k}-k^{2}\right) / 2}}{2}[s(-\mathbf{k},-\mathbf{q})+s(\mathbf{k}, \mathbf{q})-2]\right), \\
\zeta(\mathbf{k})=\int \frac{d^{2} \mathbf{q} e^{-\ell_{0}^{2} q^{2} / 2}}{(2 \pi)^{2}} V_{x z}(\mathbf{q})\left(\frac{1}{2}+\tilde{h}(\mathbf{q})+\tilde{h}(\mathbf{k})+e^{i \ell_{0}^{2} \mathbf{q} \cdot(\hat{z} \times \mathbf{k})} \tilde{h}(\mathbf{k}+\mathbf{q})\right. \\
\left.+\frac{e^{\left(q \bar{k}-k^{2}\right) / 2}}{2}[s(-\mathbf{k},-\mathbf{q})+s(\mathbf{k}, \mathbf{q})-2]\right),
\end{array}
$$

where $s\left(\mathbf{k}_{1}, \mathbf{k}_{2}\right)$ is the Fourier transform of three-point correltion function

$$
s\left(\mathbf{k}_{1}, \mathbf{k}_{2}\right)=1+\frac{1}{N} \sum_{i} \sum_{j \neq i} \sum_{n \neq j \neq i}\left\langle B_{i}\left(\mathbf{k}_{1}\right) B_{j}\left(\mathbf{k}_{2}\right) B_{n}\left(-\mathbf{k}_{1}-\mathbf{k}_{2}\right)\right\rangle_{0},
$$

and $B_{j}(\mathbf{k}) \equiv \overline{e^{i \mathbf{k} \cdot \mathbf{r}_{j}}}=e^{-\frac{|k|^{2}}{4}} \tau_{k}(j)$, which is projected free electron wave function onto LLL has been defined by Eq.(23-24) and $\langle\ldots\rangle_{0}$ is the expectation value with respect to ground state many body wave function, $\left|\Psi_{0}\right\rangle$. It is possible to find analytical expression of three-point correltion function for $\nu=1$ quantum Hall state where the many body ground state wave function may be written as a single Slater determinant [24]. The three-point correlation function in real space is 


$$
g\left(\mathbf{r}_{1}, \mathbf{r}_{2}, \mathbf{r}_{3}\right)=1-e^{-\frac{\left|z_{2}-z_{1}\right|^{2}}{2}}-e^{-\frac{\left|z_{3}-z_{2}\right|^{2}}{2}}-e^{-\frac{\left|z_{1}-z_{3}\right|^{2}}{2}}+2 e^{\frac{U}{2}} \cos \frac{V}{2}
$$

where $z_{j}=x_{j}+i y_{j}$ is in-plane complex coordinate of particles and

$$
\begin{gathered}
U=-\left(r_{1}^{2}+r_{2}^{2}+r_{3}^{2}\right)+\mathbf{r}_{1} \cdot \mathbf{r}_{2}+\mathbf{r}_{2} \cdot \mathbf{r}_{3}+\mathbf{r}_{1} \cdot \mathbf{r}_{3} \\
V=\mathbf{r}_{3} \wedge \mathbf{r}_{2}+\mathbf{r}_{2} \wedge \mathbf{r}_{1}+\mathbf{r}_{1} \wedge \mathbf{r}_{3}
\end{gathered}
$$

with $\mathbf{r}_{j} \wedge \mathbf{r}_{k}=x_{j} y_{k}-y_{j} x_{k}$

We can calculate the isomagnon dispersion relation Eq.(52) numerically after calculating realistic form-factors for SWQWs by using realistic first and second subband wave functions. We used LDFA techniques with Hedin and Lundquvist exchange-correlation energy functional in zero-magnetic field to obtain the subband wave functions. Therefore we may obtain the phase diagram for the $\nu=1$ filling factor in a SWQW structure in the relevant $d-\Delta_{S A S}$ parameter space.

In order to make a comparison with the results for DQWs [7], we associate the vanishing of the isomagnon excitation gap with the loss of incompressiblity of quantum Hall states to obtain a phase boundary for missing the odd integral QHE, as it has been defined for DQWs by MacDonald et al. [7]. In Fig.(6) and Fig.(7) we show the results of our calculation at $\nu=1$ for the isomagnon dispersion relation and the phase boundary for SWQW with different densities. At large $d$ a SWQWs may be described effectively by a DQW. In this limit the phase boundary of the SWQW is the same as a DQW with the appropriate parameters. The main difference between the DQW and SWQW phase boundaries appears when $d$ is small, which correspond to small width or density. In contrast to DQW, in SWQWs phase boundary, $d$ goes to zero where $\Delta_{S A S}$ is finite, $\Delta_{S A S}^{c}=\Delta_{S A S}(d=0)$, due to critical charge density effect where the short range component of the isopin coulomb interaction is soften and the system evolve to one-component normal state. In Fig.(7), our phase boundary is compared with DQWs and the result of LDFA in zero magnetic field for a given well width. The latter is consistent with the reported phase transition [1]. As Fig.(7) shows, the phase transition occurs at smaller $\Delta_{S A S}$ in comparison with the result of MacDonald et al. [7] 
for a given $d$. These results illustrate that our model leads to a more realistic prediction for phase trnsition in comparison with the previous models, however, our results can not capture the whole experimental datas which has been reported for phase boundary [1,2]. Note that the experimental results of Princeton group [1, 2] are based on the zero magnetic field measurements of $\Delta_{S A S}$ and calculated $d$ by LDFA.

\section{CONCLUSION}

We have presented the study of the quantum Hall effect in the single wide quantum wells. We showed that the effect of integral overlap in the middle of the SWQW has a significant effect to the quantum Hall states in these systems.

We showed that the tunneling term $\left(\Delta_{S A S}\right)$ and the interlayer separation $(d)$ are both renormalised due to the strong magnetic field. One may observe these effects experimentally. We also used and comparing different techniques, projected many body Hamiltonian onto lowest Landau levels, Hartree-Fock approximation and local density functional approximation to evaluate the tunneling term and interlayer separation. Our results confirm that the tunneling term decreases by the strong magnetic field and hence increasing the interlayer separation.

We found a phase boundary for integral quantum Hall phase transition at $\nu=1$. We showed that our model cover the most part of phase boundary which has been reportaed experimentally [2] in comparison with pervious models [7,5].

\section{ACKNOWLEDGMENTS}

It is pleasure to acknowledge useful conversation with S. M. Girvin, Song He, A. H. MacDonald, S. Rouhani and M. Shayegan. The work at Indiana University is supported by grant DMR-9416906. One of us (MA) acknowledges The Center for Thoretical Physics and Mathematics, AEOI, Tehran, Iran for the financial support. 


\section{REFERENCES}

[1] Y. W. Suen, J. Jo, M. B. Santo, L. W. Engel, S. W. Hwang and M. Shayegan, Phys. Rev. B 44, 5947 (1991). Y. W. Suen, J. Jo, M. B. Santo, and M. Shayegan, Phys. Rev. Lett. 24, 3551 (1992).

[2] Y. W. Suen, L. W. Engel, M. B. Santo, M. Shayegan and D. C. Tsui, Phys. Rev. Lett. 68, 1379 (1992). Y. W. Suen, H. C. Manoharan, X. Ying, M. B. Santos, and M. Shayegan, Phys. Rev. Lett. 72, 3405 (1994). T. S. Lay, Y. W. Suen, H. C. Manoharan, X. Ying, M. B. Santos, and M. Shayegan, Phys. Rev. B 50, 17725 (1994). H. C. Manoharan, Y. W. Suen, M. B. Santos, and M. Shayegan, preprint, cond-mat/9603008.

[3] G. S. Boebinger, H. W. Jiang, L. N. Pfeiffer, and K. W. West, Phys. Rev. Lett. 64,1793 (1990).

[4] J. P. Eisenstein, G. S. Boebinger, L. N. Pfeiffer, K. W. West, and Song He, Phys. Rev. Lett. 68, 1383 (1992).

[5] Song He, S. Das Sarma, X. C. Xie, Phys. Rev. B 47, 4394 (1993)

[6] B. I. Halperin, Helv. Phys. Acta. 56, 775, (1983).

[7] A. H. MacDonald, P. M. Platzman, and G. S. Boebinger, Phys. Rev. Lett. 65, 775 (1990).

[8] H. A. Fertig, Phys. Rev. B 40, 1087 (1989).

[9] S. M. Girvin, T. Jach, Phys. Rev. B 29, 5617 (1984).

[10] S. M. Girvin, A. H. MacDonald, P. M. Platzman, Phys. Rev. B 33, 2481 (1986).

[11] K. Yang, K. Moon, L. Zheng, H. Mori, A. H. MacDonald, S. M. Girvin, D. Yoshioka, S. Zhang, Phys. Rev. Lett 72, 732 (1994).

[12] K. Moon, H. Mori, K. Yang, S. M. Girvin, A. H. MacDonald, L. Zheng, D. Yoshioka, S. Zhang, Phys. Rev. B 51, 5138 (1995). S. M. Girvin, A. H. MacDonald, in 
Novel Quantum Liquids in Semiconductor Structures, edited by S. DasSarma and A. Pinczuk (wiley, New York, 1996).

[13] C. Kittel, Quantum Theory of Solids (Wiley, New York, 1963).

[14] S. M. Girvin, A. H. MacDonald, P. M. Platzman, Phys. Rev. B 33, 2481 (1986).

[15] S. M. Girvin, in The Quantum Hall Effect, edited by Richard E. Prang and Steven M. Girvin, (Spriger-Verlag, New York, 1987).

[16] M. Abolfath (Unpublished).

[17] Eduardo Fradkin, Field Theories of Condensed Matter Systems (Addison Wesley, 1991).

[18] F. Stern and S. Das Sarma, Phys. Rev. B 30,840 (1984).

[19] G. Vignale, M. Rasolt, Phys. Rev. B 59, 2360 (1987).

[20] S. H. Vosko, L. Wilk, M. Nusair, Can. J. Phys. 58, 1200 (1980).

[21] D. M. Ceperly, B. J. Alder, Phys. Rev. Lett. 45, 566 (1980).

[22] L. Hedin, B. I. Lundqvist, J. Phys. C 4, 2064 (1971).

[23] Xiao-Gang Wen, A. Zee, Phys. Rev. Lett. 69, 1811 (1992).

[24] B. Jancovici, Phys. Rev. Lett. 46, 386 (1981). A. H. MacDonald and S. M. Girvin, Phys. Rev. B 38, 6295 (1988).

[25] M. Shayegan, private communication. 


\section{FIGURES}

FIG. 1. Numerical calculated electron wavefunction of zero magnetic field symmetric and antisymmetric subbands of a SWQW with $W=1000(\AA)$ and $N_{s}=3.1 \times 10^{11} \mathrm{~cm}^{-2}$. The envelop wavefunction in the middle of the sample is small due to tunneling through the self consistent potential barrier of the SWQW. The symmetric and antisymmetric wave functions are close to each other and DQW is a good approximation for this case.

FIG. 2. $V_{x x}(\mathbf{q})$ (solid line) and $V_{z z}(\mathbf{q})$ (dash line) are shown for two different SWQW with $W=680(\AA), N_{s}=1.8 \times 10^{11} \mathrm{~cm}^{-2}$ Fig.(a) and $N_{s}=3.1 \times 10^{11} \mathrm{~cm}^{-2}$ Fig.(b). The $V_{0 z}(\mathbf{q})$ is supressed by increasing the areal electron density. $V_{z z}$ in (a) is scaled by 10 and in (b) by 100 to show in the above figures.

FIG. 3. Numerical results of $V_{0 z}\left(\mathbf{Q} \ell_{0}\right)$ vs $\mathbf{Q} \ell_{0}$ is shown for three different SWQW's densities with $W=680(\AA)$. Varing areal densities $N_{s}=1.8,2.3$ and $3.1 \times 10^{11} \mathrm{~cm}^{2}$ are shown by long dashed, dashed and solid curves. The inset exhibits the calculated electron wave function of zero magnetic field symmetric and antisymetric subbands of a SWQW for $N_{s}=3.1 \times 10^{11} \mathrm{~cm}^{-2}$.

FIG. 4. Numerical results of $V_{0 z}\left(\mathbf{Q} \ell_{0}\right)$ vs $\mathbf{Q} \ell_{0}$ is shown for SWQW's electron areal density $0.5 \times 10^{11} \mathrm{~cm}^{-2}$ with $W=680(\AA)$. The integral of $V_{0 z}\left(\mathbf{Q} \ell_{0}\right) e^{-Q^{2} \ell_{0}^{2} / 2}$ is small and therefore the $\Delta_{S A S}$ enhancement due to strong $B$ is small. The inset exhibits the calculated electron density along $z$-direction for two different regimes, zero magnetic field (dotted points) and strong magnetic field (dashed line) which demonstrates a quasi 3D electron system. These two densities profile are nearly the same and the effect of strong magnetic field on the charge density along the $z$-axis is negligible which is true for 3D electron system.

FIG. 5. Electron density for self-consistent calculations in zero (solid curve) and strong magnetic field (dashed curve) for $N_{s}=3.1 \times 10^{11} \mathrm{~cm}^{-2}$ and $W=680(\AA)$. The effect of strong $B$ is decreasing (increasing) the probability of finding an electron in the edge (middle) of the SWQW which means that electrons are pushed to go in the middle of SWQW by the magnetic field. 
FIG. 6. Calculated phase diagram for the $\nu=1$ QHE in SWQW system. The lines denote the boundaries of phase corresponding to compressible-incompressible transition. These calculation has done for three differnt SWQW's width 400, 680 and $800 \AA$. The phase boundary touch horizontal axis at $\Delta_{S A S}^{c}$ which corresponds to critical density $N_{s c}$ and critical width $W_{c} . \Delta_{S A S}^{c}$ decreased with increasing of $W$. This is the crossover between one layer compressible and incompressible quantum Hall systems. The solid line is the calculated phase boundary of DQW for $\delta$-layers separated by a distance $d$ which is the asymptotic limit of large SWQW's width, i.e. the behavior of phase boundary for the sample $W=800$ is closer to the DQW. The inset exhibits the isomagnon dispersion relation for a SWQWs width $W=680$ and the areal density $N_{s}=1.8 \times 10^{11} \mathrm{~cm}^{-2}$ at the phase boundary where the isomagnon gap is zero. The energy of isomagnons at zero wave vector is specified by the $\Delta_{S A S}^{B}$ where at $\nu=1$, the zero tunneling yields gapless dispersion relation.

FIG. 7. The comparison between the realistic (triangles) for a SWQW with $W=680 \AA$ width and DQW (solid line) phase diagrams are shown in this figure. The results of our zero magnetic local density functional results (circles) is shown. The integral quantum Hall phase transition has been reported in $\Delta_{S A S} /\left(e^{2} / \epsilon_{0} \ell_{0}\right) \sim 0.05$ at zero magnetic field. The results of our calcuation is closer to the experimental phase boundary than the results of DQW approximation. 


\section{TABLES}

TABLE I. Comparison of zero magnetic field $\Delta_{S A S}$ which is obtained by Hedin and Lundqvist LDFA in zero magnetic field and renormalized $\Delta_{S A S}^{B}(\nu=1), \Delta_{S A S}^{\prime B}(\nu=1)$ which is obtained by many body Hamiltonian and LDFA in the presence of strong perpendicular magnetic field and also renormalized $\Delta_{S A S}^{B}$ with filling factor $\nu=1 / 2$, for a $680(\AA)$ wide well vs areal density $N_{s}$.

\begin{tabular}{lcccc}
\hline \hline$N_{s}\left(10^{11} \mathrm{~cm}^{-2}\right)$ & $\Delta_{\text {sas }}(K)$ & $\Delta_{\text {sas }}^{B}(\nu=1)(K)$ & $\Delta_{S A S}^{B}(\nu=1)(K)$ & $\Delta_{S A S}^{B}(\nu=1 / 2)(K)$ \\
\hline 1.8 & 12.5 & 12.2 & 11.4 & 12.1 \\
2.3 & 9.5 & 8.2 & 8.5 & 7.7 \\
2.8 & 7.4 & 5.8 & 6.6 & 5.0 \\
3.1 & 6.4 & 4.7 & 5.7 & 4.0 \\
\hline \hline
\end{tabular}

